\title{
Molecular Detection of Avian Pathogenic Escherichia coli (APEC) for the First Time in Layer Farms in Bangladesh and Their Antibiotic Resistance Patterns
}

\author{
Samina Ievy, Md. Saiful Islam ${ }^{\circledR}$, Md. Abdus Sobur ${ }^{\circledR}$, Mithun Talukder, Md. Bahanur Rahman, \\ Mohammad Ferdousur Rahman Khan and Md. Tanvir Rahman * (D) \\ Department of Microbiology and Hygiene, Faculty of Veterinary Science, Bangladesh Agricultural University, \\ Mymensingh 2202, Bangladesh; v.samina@gmail.com (S.I.); dvm41257@bau.edu.bd (M.S.I.); \\ soburvetbau@gmail.com (M.A.S.); saddomithun@gmail.com (M.T.); bahanurr@bau.edu.bd (M.B.R.); \\ mfrkhan@bau.edu.bd (M.F.R.K.) \\ * Correspondence: tanvirahman@bau.edu.bd
}

Received: 3 June 2020; Accepted: 7 July 2020; Published: 9 July 2020

\begin{abstract}
Avian pathogenic Escherichia coli (APEC) causes significant economic losses in poultry industries. Here, we determined for the first time in Bangladesh, the prevalence of APEC-associated virulence genes in E. coli isolated from layer farms and their antibiotic resistance patterns. A total of 99 samples comprising internal organs, feces, and air were collected from 32 layer farms. Isolation was performed by culturing samples on eosin-methylene blue agar plates, while the molecular detection of APEC was performed by PCR, and antibiograms were performed by disk diffusion. Among the samples, 36 were positive for the APEC-associated virulence genes fimC, iucD, and papC. Out of 36 isolates, 7, 18, and 11 were positive, respectively, for three virulence genes ( virulence genes was significantly higher in the internal organs, the air and feces were also positive. The antibiograms revealed that all the isolates $(100 \%)$ were resistant to ampicillin and tetracycline; $97.2 \%$, to chloramphenicol and erythromycin; $55.5 \%$, to enrofloxacin; $50.0 \%$, to norfloxacin and ciprofloxacin; $19.4 \%$, to streptomycin; $11.1 \%$, to colistin; and $8.33 \%$, to gentamicin. Interestingly, all the isolates were multidrug-resistant (MDR). Spearman's rank correlation coefficient analysis revealed the strongest significant correlation between norfloxacin and ciprofloxacin resistance. This is the first study in Bangladesh describing the molecular detection of APEC in layer farms. Isolated APEC can now be used for detailed genetic characterization and assessing the impact on public health.
\end{abstract}

Keywords: avian pathogenic E. coli; virulence genes; multidrug-resistant; layer farm; air; public health

\section{Introduction}

Poultry farming is a well-developed and profitable agri-business in Bangladesh. It is estimated that the livestock and poultry sector contributes around $1.47 \%$ of the total GDP of the country [1]. Poultry products including eggs and meat chiefly fulfil the daily protein requirements of Bangladesh's population. However, from time to time, the advancement of poultry production is seriously hampered by various infectious diseases [2]. Among the infectious agents, avian pathogenic Escherichia coli (APEC) is thought to be prevalent in Bangladesh. Although E. coli is part of the normal bacterial microbiota of the intestinal tract, other mucosal surfaces of hosts, and the poultry farm environment, few of these strains are endowed with specific virulence factors that define the APEC phenotype [3]. Most APEC strains are phylogenetically associated with extra-intestinal location [4,5]. Principally, 
they are associated with respiratory tract and systemic infections [6]. In addition, APEC respiratory infections are secondary to other respiratory tract infections, including infectious bronchitis virus (avian coronavirus), Newcastle disease virus, and Mycoplasma gallisepticum [7].

Avian colibacillosis, caused by APEC, is a complex syndrome that has an ominous impact on the poultry sector worldwide [8]. Avian colibacillosis is characterized by multiple organ lesions such as air sacculitis, pericarditis, peritonitis, salpingitis, synovitis, osteomyelitis, yolk sac infections, etc. $[9,10]$. Thus, APEC is a major cause of extensive economic loss in the poultry industry due to high morbidity and mortality [4]. Both the broiler and layer farms are affected by APEC. Several of the characteristic virulence associated genes (VAGs) of APEC are iss, $k p s, c v i$, tss, papC, fim $C$, iucD, etc. [6,11]. Virulence factors (invasins, adhesins, iron acquisition systems, toxins, and protectins) coded by multifarious VAGs facilitate the infection-causing abilities of $E$. coli strains [8]. These virulence factors may become useful for pathogenic strains of E. coli by enabling invasion, colonization, and adherence and protecting E. coli from host defenses [12-14]. Among them, papC (pyelonefritis associated to pili C) is associated with the production of adhesion-related factors to enable the adhesion of $E$. coli and is responsible for occurring infections [8,15], fim C (Type 1 fimbriae C) is connected with the adhesion and colonization of E. coli on epithelial cells [16], and iucD (iron-uptake systems of E. coli $\mathrm{D}$ ) demonstrates the difference between APEC and non-APEC isolates in terms of the iron acquisition system [17]. The virulence of any particular isolate of E. coli correlates with the number and combination of these virulence-associated genes [18]. These virulence factors may be found as single genes or as associations of different gene combinations in both healthy and clinical isolates [19].

Along with APEC, uropathogenic E. coli (UPEC) and neonatal meningitis E. coli (NMEC) are also considered as extra-intestinal pathogenic E. coli (ExPEC). In humans, ExPEC may cause urinary tract infections (UTIs), neonatal meningitis, and septicemia [20,21]. Phylogenic similarities among APEC, UPEC, and NMEC strains-transmissible plasmids, virulence genes, and other genetic characteristics-indicate that APEC are zoonotic in nature [21,22]. In addition, APEC are present in the intestines and meat of healthy poultry, possessing genetic similarities with human ExPEC, indicating a possible transmission of animal APEC to humans [22]. Some experimental studies have expounded the transmission pattern between avian ExPEC and human ExPEC with a negative impact on public health [23].

Antimicrobial resistance (AMR) is an ever-increasing public health crisis. The G20 partners have recognized AMR as a major "growing threat to public health and economic growth". It causes an estimated 700,000 deaths each year across the world [24]. Drug-resistant APEC strains can contaminate the food supply from farm to fork through eggs, meat, and other contaminants and thus pose a severe threat to the consumer's health [25]. The indiscriminate use of antibiotics in poultry production may have contributed to drug resistance in APEC. From the poultry farm, drug-resistant strains are deposited into soil, wastewater, air, and the environment [26].

Studies from many countries have detected drug resistance determinants in APEC [27,28]. In Bangladesh, previous studies have identified antibiotic-resistant E. coli from poultry [29-32]. These studies, however, did not focus on APEC and the associated virulence genes. Therefore, the present study was designed to determine the prevalence of APEC and the associated virulence genes in E. coli in layer farms in Bangladesh, as well as their antibiotic resistance profile.

\section{Materials and Methods}

\subsection{Ethics Statement}

The experimental procedures and protocols used in this study were approved by the Animal Welfare and Experimentation Ethics Committee of Bangladesh Agricultural University (approval number AWEEC/BAU/2019(28)). 


\subsection{Sample Collection and Processing}

Samplings were done in January-November 2019 from 32 layer farms located in Mymensingh district, Bangladesh. A total of 99 samples were collected aseptically, comprising seven different types of samples, including air from the insides of poultry shades $(n=31)$, feces from sick birds $(n=32)$, and the intestinal organs (trachea, intestine, liver, lung, and egg yolk material; $n=36$ ) of dead birds. Air sampling was done using the settle plate method as previously described by other [33] with some modifications. In brief, instead of nutrient agar, here, eosin methylene blue (EMB) agar plates were exposed at $1 \mathrm{~m}$ above the ground to different corners of the poultry shades for $10 \mathrm{~min}$. Freshly dropped fecal samples were collected using sterile cotton buds from sick isolated groups of birds. Internal organs were collected during post-mortem examinations. All the collected samples were given unique tag numbers and transported to the laboratory maintaining the cold chain. Immediately after arrival at the laboratory, fecal samples $(1 \mathrm{~g})$ were seeded into test tubes containing $5 \mathrm{~mL}$ of nutrient broth. Internal organs were initially cut into small pieces and then transferred into test tubes containing $5 \mathrm{~mL}$ of nutrient broth. EMB agar plates and test tubes were then incubated aerobically at $37^{\circ} \mathrm{C}$ overnight.

\subsection{Isolation and Identification of E. coli}

The isolation and identification of E. coli was based on culture on EMB agar plates. For this purpose, the overnight-grown broth cultures were streaked on EMB agar plates and incubated aerobically at $37^{\circ} \mathrm{C}$ overnight. Single metallic sheen colonies on the EMB agar plates were considered as indicative of E. coli. These colonies were then subjected to morphological study by Gram staining, basic sugar fermentation tests, methyl red tests, Voges-Proskauer tests, and indole tests [34]. The final confirmation of the isolation of E. coli was performed by polymerase chain reactions (PCRs) targeting the E. coli $16 \mathrm{~S}$ rRNA gene [35].

For PCR, genomic DNA was extracted from E. coli pure cultures by the boiling method [36]. In brief, a pure colony was put into an Eppendorf tube containing $100 \mu \mathrm{L}$ of deionized water and gently vortexed, followed by boiling and cooling for 10 min during each step. Finally, genomic DNA was collected after centrifugation for $10 \mathrm{~min}$ and stored at $-20^{\circ} \mathrm{C}$ for further use.

\subsection{Molecular Detection of APEC-Associated Virulence Genes}

Several genes are known to be associated specifically with APEC. In this study, we selected the fim $C$, $i u c D$, and $p a p C$ genes as the molecular markers for the detection of APEC. These are the commonly APEC-associated virulence genes detected in the majority of the studies focused on APEC $(8,22,60,61,62,74)$. Moreover, as mentioned earlier, iucD differentiates APEC from non-APEC (17). Once confirmed, isolated E. coli were screened by PCR for detecting the APEC-associated virulence genes fim $C$, iucD, and papC [12]. The primers used for the detection of APEC are listed in Table 1.

Table 1. Primers used in the detection of avian pathogenic Escherichia coli (APEC)-associated virulence genes.

\begin{tabular}{|c|c|c|c|c|}
\hline Target Genes & Primer Sequence $\left(5^{\prime}-3^{\prime}\right)$ & $\begin{array}{l}\text { Amplicon } \\
\text { Size (bp) }\end{array}$ & $\begin{array}{c}\text { Annealing } \\
\text { Temperature }\left({ }^{\circ} \mathrm{C}\right)\end{array}$ & References \\
\hline $16 \mathrm{~S}$ rRNA & $\begin{array}{l}\text { F: GACCTCGGTTTAGTTCACAGA } \\
\text { R: CACACGCTGACGCTGACCA }\end{array}$ & 585 & 55 & [35] \\
\hline fimC & $\begin{array}{l}\text { F: GGGTAGAAAATGCCGATGGTG } \\
\text { R: CGTCATTTTGGGGGTAAGTGC }\end{array}$ & 496 & 59 & \multirow{3}{*}{ [12] } \\
\hline$i u c D$ & $\begin{array}{l}\text { F: ACAAAAAGTTCTATCGCTTCC } \\
\text { R: CCTGATCCAGCTGATGCTC }\end{array}$ & 692 & 55 & \\
\hline papC & $\begin{array}{l}\text { F: TGATATCACGCAGTCAGTAGC } \\
\text { R: CCGGCCATATTCACATAA }\end{array}$ & 483 & 59 & \\
\hline
\end{tabular}


PCR tests were done in a final $25 \mu \mathrm{L}$ reaction with $12.5 \mu \mathrm{L}$ of master mix (2X) (Promega, Madison, WI, USA), $4 \mu \mathrm{L}$ of genomic DNA (50 ng/ $\mu \mathrm{L}$ ), $1 \mu \mathrm{L}$ of each primer, and $6.5 \mu \mathrm{L}$ of nuclease-free water. After completion, the amplified PCR products were analyzed by electrophoresis in $1.5 \%$ agarose. Amplicons were stained by ethidium bromide and visualized under an ultraviolet trans-illuminator (Biometra, Göttingen, Germany). A 100 bp DNA ladder (Promega, Madison, WI, USA) was used to check the size of the PCR amplicons.

\subsection{Antimicrobial Susceptibility Test}

Isolated E. coli positive for APEC-associated virulence genes were used for disk diffusion tests as reported [37]. Ten commonly used antibiotics of different classes were employed: penicillins (ampicillin-2 $\mu \mathrm{g}$ disk), amphenicols (chloramphenicol-10 $\mu \mathrm{g}$ ), fluoroquinolones (ciprofloxacin $-5 \mu \mathrm{g}$; enrofloxacin-10 $\mathrm{g}$; and norfloxacin-10 $\mu \mathrm{g}$ ), polypeptides (colistin-10 $\mu \mathrm{g}$ ), macrolides (erythromycin-15 $\mu \mathrm{g}$ ), aminoglycosides (gentamicin-10 $\mu \mathrm{g}$; and streptomycin-10 $\mu \mathrm{g}$ ), and tetracycline (tetracycline-30 $\mu \mathrm{g}$ ). Antimicrobial susceptibility tests (ASTs) were performed on Mueller-Hinton agar plates (Himedia, India) with a concentration of freshly grown bacteria equal to $0.5 \mathrm{McFarland}$ units. The results were recorded as sensitive, intermediate, or resistant as per the standards of the Clinical and Laboratory Standards Institute [38]. Multidrug-resistant (MDR) isolates were categorized according to Sweeney et al. [39].

\subsection{Statistical Analysis}

The results were inserted into an Excel 2013 spreadsheet (Microsoft Office 2013, Microsoft, Los Angeles, CA, USA) and analyzed using the SPSS software (IBM SPSS version 25.0, IBM, Chicago, IL, USA). Descriptive analysis was conducted to calculate prevalence. A chi-square test for relatedness was done to determine the possible relationships of the sample type with the prevalence of $E$. coli and APEC-associated virulence genes. The chi-square test for goodness-of-fit was applied to observe if any differences existed among the frequencies of the three APEC-associated virulence genes. A $p$-value less than 0.05 ( $p$-value $<0.05$ ) was considered as statistically significant.

In addition, a Spearman rank correlation with a Bonferroni correction $(\alpha / 8)$ was performed to determine the possible pairwise correlation among various antimicrobial resistance patterns using a piece of statistical software named STATA (STATA version 16.0) as previously described by Varga et al. [40]. A $p$-value less than or equal to $0.00625(p \leq 0.00625 ; \alpha / 8)$ indicated the test result as statistically significant.

\section{Results}

\subsection{Prevalence of E. coli Isolates}

Among 99 samples, 82 (82.83\%) were positive for E. coli according to the PCR targeting of the E. coli $16 \mathrm{~S}$ rRNA gene. The highest prevalence was found in feces (100\%), and the lowest, in air samples (67.74\%). The overall prevalence of $E$. coli in various samples is presented in Table 2. Statistical analysis revealed that feces carried a significantly higher percentage of E. coli than the other samples investigated (chi-square test, 95\% CI, $p=0.003$ ).

Table 2. Prevalence of E. coli in layer farms.

\begin{tabular}{|c|c|c|c|c|c|c|}
\hline $\begin{array}{c}\text { Sample Source/Nature } \\
\text { of Sample }\end{array}$ & $\begin{array}{c}\text { No. of } \\
\text { Samples } \\
\text { Analyzed }\end{array}$ & $\begin{array}{c}\text { No. } \\
\text { Overall } \\
\text { Analyzed }\end{array}$ & $\begin{array}{l}\text { No. E. coli } \\
\text { Positive } \\
\text { Samples }\end{array}$ & $\begin{array}{c}\text { Overall } \\
\text { Positive } \\
\text { for E. coli }\end{array}$ & $\begin{array}{c}\text { Prevalence } \\
(\%)\end{array}$ & $p$-Value \\
\hline Air & 31 & 31 & 21 & 21 & 67.74 & \\
\hline Feces & 32 & 32 & 32 & 32 & 100 & \\
\hline
\end{tabular}


Table 2. Cont.

\begin{tabular}{|c|c|c|c|c|c|c|c|}
\hline \multicolumn{2}{|c|}{$\begin{array}{c}\text { Sample Source/Nature } \\
\text { of Sample }\end{array}$} & $\begin{array}{c}\text { No. of } \\
\text { Samples } \\
\text { Analyzed }\end{array}$ & $\begin{array}{c}\text { No. } \\
\text { Overall } \\
\text { Analyzed }\end{array}$ & $\begin{array}{l}\text { No. E. coli } \\
\text { Positive } \\
\text { Samples }\end{array}$ & $\begin{array}{c}\text { Overall } \\
\text { Positive } \\
\text { for E. coli }\end{array}$ & $\begin{array}{l}\text { Prevalence } \\
\text { (\%) }\end{array}$ & \multirow[t]{6}{*}{$p$-Value } \\
\hline \multirow{5}{*}{$\begin{array}{l}\text { Internal } \\
\text { organs }\end{array}$} & Trachea & 5 & \multirow{5}{*}{36} & 3 & \multirow{5}{*}{29} & \multirow{5}{*}{80.56} & \\
\hline & Intestine & 8 & & 7 & & & \\
\hline & Liver & 14 & & 11 & & & \\
\hline & Lung & 7 & & 6 & & & \\
\hline & Egg Yolk & 2 & & 2 & & & \\
\hline \multicolumn{2}{|c|}{ Total } & 99 & 99 & 82 & 82 & 82.83 & \\
\hline
\end{tabular}

\subsection{APEC-Associated Virulence Genes}

Out of 82 E. coli, 36 (36.36\%) were positive for APEC-associated virulence genes (Table 3). A significantly higher prevalence of APEC-associated virulence genes was observed in the internal organs than in air (16.13\%) and feces (21.87\%) (chi-square test, 95\% CI, $p<0.001)$.

Table 3. Virulence genes in the isolated APEC.

\begin{tabular}{|c|c|c|c|c|c|c|c|}
\hline \multirow{2}{*}{\multicolumn{2}{|c|}{ Samples }} & \multirow{2}{*}{$\begin{array}{c}\text { Name of } \\
\text { Positive Isolate }\end{array}$} & \multicolumn{3}{|c|}{$\begin{array}{l}\text { APEC-Associated } \\
\text { Virulence Genes }\end{array}$} & \multirow{2}{*}{$\begin{array}{l}\text { No. of Positive } \\
\text { Isolates (\%) }\end{array}$} & \multirow[t]{2}{*}{$p$-Value } \\
\hline & & & $\mathrm{fimC}$ & $i u c D$ & papC & & \\
\hline \multirow{5}{*}{\multicolumn{2}{|c|}{$\operatorname{Air}(n=31)$}} & A1 & + & + & + & \multirow{5}{*}{$5(16.13)$} & \multirow{21}{*}{$<0.001$} \\
\hline & & A2 & + & - & + & & \\
\hline & & A3 & + & + & + & & \\
\hline & & A4 & + & + & + & & \\
\hline & & A5 & + & + & + & & \\
\hline \multirow{7}{*}{\multicolumn{2}{|c|}{ Feces $(n=32)$}} & $\mathrm{F} 2$ & + & + & - & & \\
\hline & & F3 & + & + & + & & \\
\hline & & $\mathrm{F} 4$ & + & - & + & & \\
\hline & & F5 & + & - & + & $7(21.87)$ & \\
\hline & & F6 & + & + & + & & \\
\hline & & F7 & + & - & - & & \\
\hline & & F9 & - & - & + & & \\
\hline \multirow{9}{*}{$\begin{array}{c}\text { Internal } \\
\text { organs }(n=36)\end{array}$} & Trachea & Io-T2 & + & + & - & \multirow{9}{*}{$24(66.67)$} & \\
\hline & $(n=5)$ & Io-T3 & + & + & + & & \\
\hline & \multirow{7}{*}{$\begin{array}{l}\text { Intestine } \\
(n=8)\end{array}$} & Io-I2 & + & + & - & & \\
\hline & & Io-I3 & + & + & - & & \\
\hline & & Io-I4 & + & - & - & & \\
\hline & & Io-I5 & + & + & - & & \\
\hline & & Io-I6 & + & + & - & & \\
\hline & & Io-I7 & + & - & + & & \\
\hline & & Io-I8 & + & - & - & & \\
\hline
\end{tabular}


Table 3. Cont.

\begin{tabular}{|c|c|c|c|c|c|c|c|}
\hline \multicolumn{2}{|c|}{ Samples } & \multirow{2}{*}{$\begin{array}{c}\text { Name of } \\
\text { Positive Isolate }\end{array}$} & \multicolumn{3}{|c|}{$\begin{array}{l}\text { APEC-Associated } \\
\text { Virulence Genes }\end{array}$} & \multirow{2}{*}{$\begin{array}{l}\text { No. of Positive } \\
\text { Isolates (\%) }\end{array}$} & \multirow[t]{2}{*}{$p$-Value } \\
\hline & & & fimC & iucD & papC & & \\
\hline \multirow{15}{*}{$\begin{array}{c}\text { Internal } \\
\text { organs }(n=36)\end{array}$} & \multirow{9}{*}{$\begin{array}{l}\text { Liver } \\
(n=14)\end{array}$} & Io-L1 & + & + & - & \multirow{15}{*}{$24(66.67)$} & \multirow{15}{*}{$<0.001$} \\
\hline & & Io-L2 & + & + & - & & \\
\hline & & Io-L3 & + & - & - & & \\
\hline & & Io-L4 & + & - & - & & \\
\hline & & Io-L6 & + & - & - & & \\
\hline & & Io-L7 & + & - & - & & \\
\hline & & Io-L9 & + & + & - & & \\
\hline & & Io-L10 & + & + & - & & \\
\hline & & Io-L13 & + & + & - & & \\
\hline & \multirow{4}{*}{$\begin{array}{l}\text { Lung } \\
(n=7)\end{array}$} & Io-Lu1 & + & - & - & & \\
\hline & & Io-Lu2 & + & - & - & & \\
\hline & & Io-Lu5 & + & - & - & & \\
\hline & & Io-Lu7 & + & + & - & & \\
\hline & \multirow{2}{*}{$\begin{array}{c}\text { Egg Yolk } \\
(n=2)\end{array}$} & Io-Y1 & + & + & - & & \\
\hline & & Io-Y2 & + & + & - & & \\
\hline \multicolumn{2}{|c|}{ Total $(n=99)$} & 36 & $\begin{array}{c}35 \\
(97.22 \%)\end{array}$ & $\begin{array}{c}21 \\
(58.33 \%)\end{array}$ & $\begin{array}{c}12 \\
(33.33 \%)\end{array}$ & $36(36.36 \%)$ & \\
\hline & $p$-value & & & 0.003 & & & \\
\hline
\end{tabular}

A, Air; F, Feces; Io, Internal organs; T, Trachea; I, Intestine; L, Liver; Lu, Lung; Y, Egg yolk.

Among the 36 E. coli isolates carrying APEC-associated virulence genes, seven were positive for three virulence genes $(f i m C$, $i u c D$, and pap $)$ ); 18 were positive for two virulence genes (in different combinations), and 11 were carrying a single virulence gene (Table 3 ). The most prevalent combination was fim C/iucD (in 11 isolates); however, fim C/papC was in three isolates. Statistical analysis indicated that fimC (97.22\%) was significantly more prevalent than iucD (58.33\%) and papC (33.33\%) in 36 E. coli (chi-square test, 95\% CI, $p=0.003$ ).

\subsection{Antibiogram Profile of E. coli Isolates Carrying APEC-Associated Virulence Genes}

An antibiogram study showed that all $36 \mathrm{E}$. coli isolates carrying APEC-associated virulence genes were resistant to ampicillin and tetracycline (100\%), followed by resistance to chloramphenicol and erythromycin (97.2\%), to enrofloxacin (55.5\%), to norfloxacin and ciprofloxacin (50.0\%), to streptomycin $(19.4 \%)$, to colistin $(11.1 \%)$, and to gentamicin (8.3\%) (Figure 1). Detailed results of the AST and sample-wise antibiotic resistance profile of APEC are presented in Table 4 and Figure 2, respectively. All the APEC isolates were MDR in nature (Table 5).

Table 4. Overall results of antibiotic sensitivity tests of APEC isolates.

\begin{tabular}{cccccccccccc}
\hline SL No. & Isolate Name & CL & CIP & EX & NX & AMP & TE & S & GEN & C & E \\
\hline 1 & $\mathrm{~A} 1$ & S & S & S & S & R & R & S & S & R & R \\
2 & A2 & S & S & S & S & R & R & I & I & R & R \\
3 & A3 & S & R & R & R & R & R & R & S & R & R \\
4 & $\mathrm{~A} 4$ & $\mathrm{~S}$ & $\mathrm{R}$ & $\mathrm{R}$ & $\mathrm{R}$ & $\mathrm{R}$ & $\mathrm{R}$ & $\mathrm{R}$ & $\mathrm{S}$ & $\mathrm{R}$ & $\mathrm{R}$ \\
5 & $\mathrm{~A} 5$ & $\mathrm{~S}$ & $\mathrm{~S}$ & $\mathrm{I}$ & $\mathrm{S}$ & $\mathrm{R}$ & $\mathrm{R}$ & $\mathrm{S}$ & $\mathrm{S}$ & $\mathrm{R}$ & $\mathrm{R}$ \\
6 & $\mathrm{~F} 2$ & $\mathrm{~S}$ & $\mathrm{R}$ & $\mathrm{R}$ & $\mathrm{R}$ & $\mathrm{R}$ & $\mathrm{R}$ & $\mathrm{S}$ & $\mathrm{R}$ & $\mathrm{R}$ & $\mathrm{R}$ \\
\hline
\end{tabular}


Table 4. Cont.

\begin{tabular}{|c|c|c|c|c|c|c|c|c|c|c|c|}
\hline SL No. & Isolate Name & CL & CIP & EX & NX & AMP & TE & $S$ & GEN & $\mathrm{C}$ & E \\
\hline 7 & F3 & $S$ & $\mathrm{R}$ & $\mathrm{R}$ & $\mathrm{R}$ & $\mathrm{R}$ & $\mathrm{R}$ & $\mathrm{R}$ & $\mathrm{S}$ & $\mathrm{R}$ & $\mathrm{R}$ \\
\hline 8 & $\mathrm{~F} 4$ & S & $\mathrm{R}$ & $\mathrm{R}$ & $\mathrm{R}$ & $\mathrm{R}$ & $\mathrm{R}$ & I & $S$ & $\mathrm{R}$ & $\mathrm{R}$ \\
\hline 9 & F5 & S & S & I & S & $\mathrm{R}$ & $\mathrm{R}$ & $\mathrm{R}$ & $S$ & $\mathrm{R}$ & $\mathrm{R}$ \\
\hline 10 & F6 & S & S & I & S & $\mathrm{R}$ & $\mathrm{R}$ & $\mathrm{R}$ & $S$ & $\mathrm{R}$ & $\mathrm{R}$ \\
\hline 11 & F7 & S & S & S & S & $\mathrm{R}$ & $\mathrm{R}$ & S & S & $\mathrm{R}$ & $\mathrm{R}$ \\
\hline 12 & F9 & $S$ & S & S & $\mathrm{R}$ & $\mathrm{R}$ & $\mathrm{R}$ & $S$ & S & $\mathrm{R}$ & $\mathrm{R}$ \\
\hline 13 & Io-T2 & S & S & S & S & $\mathrm{R}$ & $\mathrm{R}$ & $S$ & I & $\mathrm{R}$ & $\mathrm{R}$ \\
\hline 14 & Io-T3 & S & $\mathrm{R}$ & $\mathrm{R}$ & $\mathrm{R}$ & $\mathrm{R}$ & $\mathrm{R}$ & $\mathrm{R}$ & $S$ & $\mathrm{R}$ & $\mathrm{R}$ \\
\hline 15 & Io-I2 & S & $\mathrm{R}$ & $\mathrm{R}$ & $\mathrm{R}$ & $\mathrm{R}$ & $\mathrm{R}$ & S & $S$ & $\mathrm{R}$ & $\mathrm{R}$ \\
\hline 16 & Io-I3 & S & $\mathrm{R}$ & $\mathrm{R}$ & $\mathrm{R}$ & $\mathrm{R}$ & $\mathrm{R}$ & S & S & $\mathrm{R}$ & $\mathrm{R}$ \\
\hline 17 & Io-I4 & $S$ & I & $\mathrm{R}$ & I & $\mathrm{R}$ & $\mathrm{R}$ & $S$ & S & $\mathrm{R}$ & $\mathrm{R}$ \\
\hline 18 & Io-I5 & $\mathrm{R}$ & $\mathrm{R}$ & $\mathrm{R}$ & I & $\mathrm{R}$ & $\mathrm{R}$ & $S$ & $\mathrm{R}$ & $\mathrm{R}$ & $\mathrm{R}$ \\
\hline 19 & Io-I6 & $S$ & $S$ & $\mathrm{R}$ & $S$ & $\mathrm{R}$ & $\mathrm{R}$ & $S$ & $S$ & $\mathrm{R}$ & $\mathrm{R}$ \\
\hline 20 & Io-I7 & $S$ & I & $\mathrm{R}$ & $\mathrm{R}$ & $\mathrm{R}$ & $\mathrm{R}$ & $S$ & $\mathrm{R}$ & $\mathrm{R}$ & $\mathrm{R}$ \\
\hline 21 & Io-I8 & $S$ & $\mathrm{R}$ & S & $S$ & $\mathrm{R}$ & $\mathrm{R}$ & S & S & $\mathrm{R}$ & $\mathrm{R}$ \\
\hline 22 & Io-L1 & $\mathrm{R}$ & $\mathrm{R}$ & $\mathrm{R}$ & $\mathrm{R}$ & $\mathrm{R}$ & $\mathrm{R}$ & $S$ & S & $\mathrm{R}$ & $\mathrm{R}$ \\
\hline 23 & Io-L2 & $S$ & $\mathrm{R}$ & $\mathrm{R}$ & $\mathrm{R}$ & $\mathrm{R}$ & $\mathrm{R}$ & $S$ & $S$ & $\mathrm{R}$ & $\mathrm{R}$ \\
\hline 24 & Io-L3 & $S$ & $\mathrm{R}$ & $\mathrm{R}$ & $\mathrm{R}$ & $\mathrm{R}$ & $\mathrm{R}$ & I & $S$ & $\mathrm{R}$ & I \\
\hline 25 & Io-L4 & S & $S$ & $S$ & $S$ & $\mathrm{R}$ & $\mathrm{R}$ & I & $S$ & $\mathrm{R}$ & $\mathrm{R}$ \\
\hline 26 & Io-L6 & $S$ & $S$ & $S$ & $S$ & $\mathrm{R}$ & $\mathrm{R}$ & $S$ & $S$ & $\mathrm{R}$ & $\mathrm{R}$ \\
\hline 27 & Io-L7 & $S$ & $S$ & $S$ & $S$ & $\mathrm{R}$ & $\mathrm{R}$ & $S$ & $S$ & $\mathrm{R}$ & $\mathrm{R}$ \\
\hline 28 & Io-L9 & $S$ & S & S & $S$ & $\mathrm{R}$ & $\mathrm{R}$ & S & $S$ & $\mathrm{R}$ & $\mathrm{R}$ \\
\hline 29 & Io-L10 & $S$ & $\mathrm{R}$ & $\mathrm{R}$ & $\mathrm{R}$ & $\mathrm{R}$ & $\mathrm{R}$ & $S$ & S & I & $\mathrm{R}$ \\
\hline 30 & Io-L13 & $S$ & $\mathrm{R}$ & $\mathrm{R}$ & $\mathrm{R}$ & $\mathrm{R}$ & $\mathrm{R}$ & $S$ & S & $\mathrm{R}$ & $\mathrm{R}$ \\
\hline 31 & Io-Lu1 & $S$ & $\mathrm{R}$ & $\mathrm{R}$ & $\mathrm{R}$ & $\mathrm{R}$ & $\mathrm{R}$ & I & $S$ & $\mathrm{R}$ & $\mathrm{R}$ \\
\hline 32 & Io-Lu2 & $\mathrm{R}$ & $\mathrm{R}$ & $\mathrm{R}$ & $\mathrm{R}$ & $\mathrm{R}$ & $\mathrm{R}$ & $\mathrm{R}$ & $S$ & $\mathrm{R}$ & $\mathrm{R}$ \\
\hline 33 & Io-Lu5 & $S$ & S & S & $\mathrm{S}$ & $\mathrm{R}$ & $\mathrm{R}$ & S & S & $\mathrm{R}$ & $\mathrm{R}$ \\
\hline 34 & Io-Lu7 & $S$ & I & I & $S$ & $\mathrm{R}$ & $\mathrm{R}$ & $S$ & $S$ & $\mathrm{R}$ & $\mathrm{R}$ \\
\hline 35 & Io-Y1 & $S$ & $\mathrm{R}$ & $\mathrm{R}$ & $\mathrm{R}$ & $\mathrm{R}$ & $\mathrm{R}$ & $S$ & S & $\mathrm{R}$ & $\mathrm{R}$ \\
\hline 36 & Io-Y2 & $\mathrm{R}$ & $S$ & I & $S$ & $\mathrm{R}$ & $\mathrm{R}$ & $S$ & S & $\mathrm{R}$ & $\mathrm{R}$ \\
\hline
\end{tabular}

A, Air; F, Feces; Io, Internal organs; T, Trachea; I, Intestine; L, Liver; Lu, Lung; Y, Egg yolk; CL, Colistin; CIP, Ciprofloxacin; EX, Enrofloxacin; NX, Norfloxacin; AMP, Ampicillin; TE, Tetracycline; S, Streptomycin; GEN, Gentamicin; C, Chloramphenicol; E, Erythromycin.

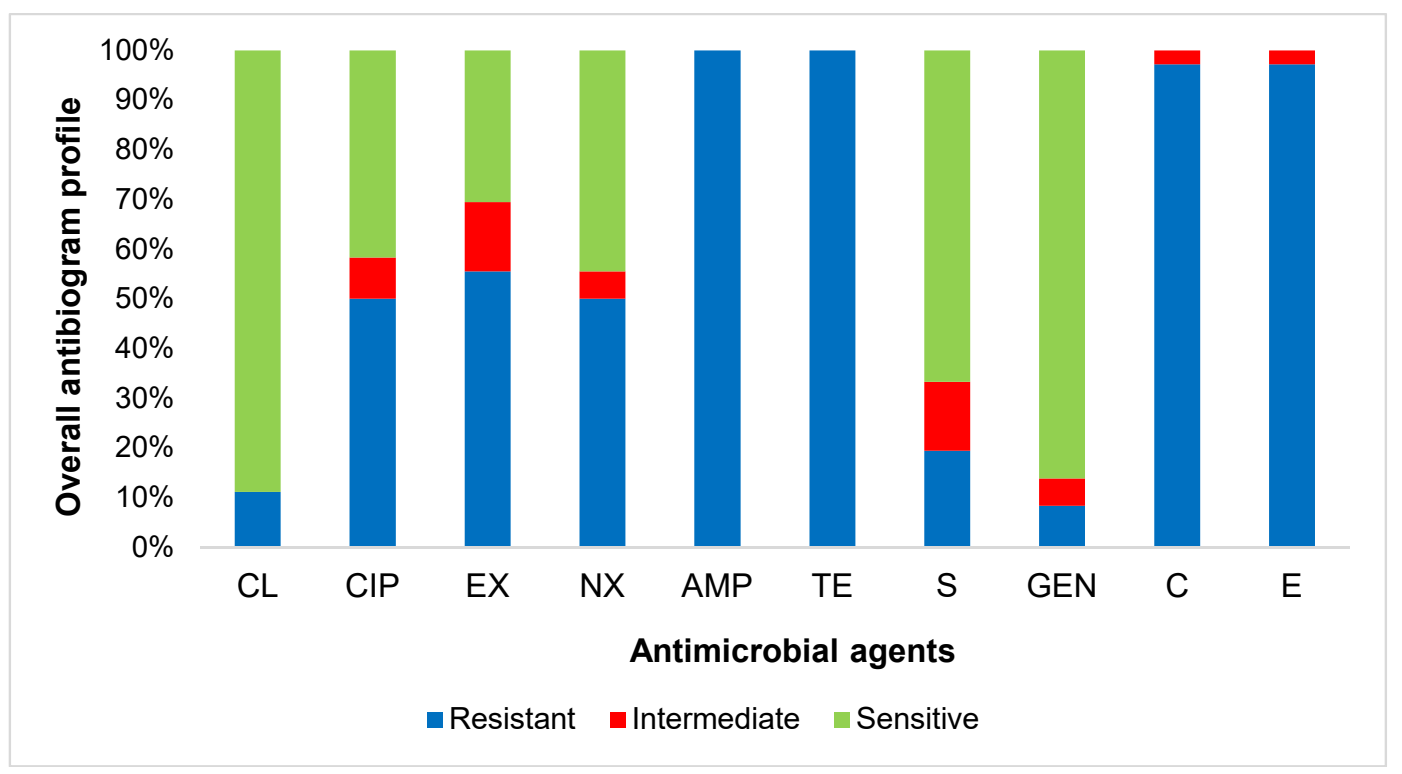

Figure 1. Overall antibiogram profile of the isolated APEC. CL, Colistin; CIP, Ciprofloxacin; EX, Enrofloxacin; NX, Norfloxacin; AMP, Ampicillin; TE, Tetracycline; S, Streptomycin; GEN, Gentamicin; C, Chloramphenicol; E, Erythromycin. 


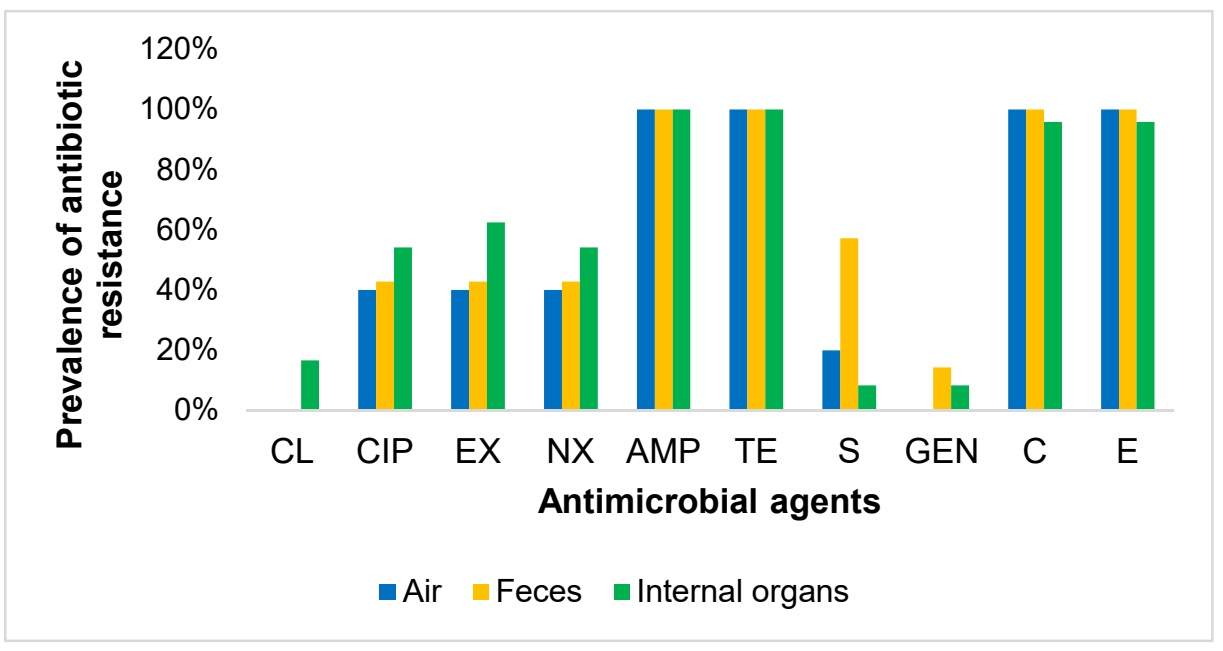

Figure 2. Sample wise antibiotic resistance profile of the isolated APEC. CL, Colistin; CIP, Ciprofloxacin; EX, Enrofloxacin; NX, Norfloxacin; AMP, Ampicillin; TE, Tetracycline; S, Streptomycin; GEN, Gentamicin; C, Chloramphenicol; E, Erythromycin.

Table 5. Multidrug resistance profile of the isolated APEC.

\begin{tabular}{|c|c|c|c|c|}
\hline Pattern No. & $\begin{array}{c}\text { Antibiotic Resistance } \\
\text { Pattern }\end{array}$ & $\begin{array}{l}\text { No. of Antibiotics } \\
\text { (Classes) }\end{array}$ & Isolate No. & $\begin{array}{c}\text { No. of } \\
\text { Isolates (\%) }\end{array}$ \\
\hline 1 & $\mathrm{AMP}, \mathrm{TE}, \mathrm{C}, \mathrm{E}$ & $4(4)$ & $\begin{array}{c}\text { A2, A5, F6, F7, Io-T2, } \\
\text { Io-L4, Io-L6, Io-L7, Io-L9, } \\
\text { Io-Lu5, Io-Lu7 }\end{array}$ & $11(30.55)$ \\
\hline 2 & AMP, TE, S, C, E & $5(5)$ & F5, F6 & $2(5.55)$ \\
\hline 3 & $\mathrm{CL}, \mathrm{AMP}, \mathrm{TE}, \mathrm{C}, \mathrm{E}$ & $5(5)$ & Io-Y2 & $1(2.78)$ \\
\hline 4 & EX, AMP, TE, C, E & $5(5)$ & Io-I4, Io-I6 & $2(5.55)$ \\
\hline 5 & NX, AMP, TE, C, E & $5(5)$ & F9 & $1(2.78)$ \\
\hline 6 & CIP, AMP, TE, C, E & $5(5)$ & Io-I8 & $1(2.78)$ \\
\hline 7 & CIP, EX, NX, AMP, TE, E & $6(4)$ & Io-L10 & $1(2.78)$ \\
\hline 8 & CIP, EX, NX, AMP, TE, C & $6(4)$ & Io-L3 & $1(2.78)$ \\
\hline 9 & CIP, EX, NX, AMP, TE, C, E & $7(5)$ & $\begin{array}{l}\text { F4, Io-L2, Io-Y1, Io-I2, } \\
\text { Io-I3, Io-L13 }\end{array}$ & $6(16.67)$ \\
\hline 10 & EX, NX, AMP, TE, GEN, C, E & $7(6)$ & Io-I7 & $1(2.78)$ \\
\hline 11 & CIP, EX, NX, AMP, TE, C, E & $7(5)$ & Io-Lu1 & $1(2.78)$ \\
\hline 12 & $\begin{array}{c}\mathrm{CIP}, \mathrm{EX}, \mathrm{NX}, \mathrm{AMP}, \mathrm{TE}, \mathrm{S}, \\
\text { C, E }\end{array}$ & $8(6)$ & A3, A4, F3, Io-T3 & $4(11.11)$ \\
\hline 13 & $\begin{array}{c}\mathrm{CL}, \mathrm{CIP}, \mathrm{EX}, \mathrm{AMP}, \mathrm{TE}, \mathrm{GEN}, \\
\mathrm{C}, \mathrm{E}\end{array}$ & $8(7)$ & Io-I5 & $1(2.78)$ \\
\hline 14 & $\begin{array}{c}\text { CIP, EX, NX, AMP, TE, GEN, } \\
\text { C, E }\end{array}$ & $8(6)$ & $\mathrm{F} 2$ & $1(2.78)$ \\
\hline 15 & $\begin{array}{c}\mathrm{CL}, \mathrm{CIP}, \mathrm{EX}, \mathrm{NX}, \mathrm{AMP}, \mathrm{TE}, \\
\mathrm{C}, \mathrm{E}\end{array}$ & $8(6)$ & Io-L1 & $1(2.78)$ \\
\hline \multirow[t]{2}{*}{16} & $\begin{array}{c}\text { CL, CIP, EX, NX, AMP, TE, S, } \\
\text { C, E }\end{array}$ & $9(7)$ & Io-Lu2 & $1(2.78)$ \\
\hline & Total & & & 36 \\
\hline
\end{tabular}

CL, Colistin; CIP, Ciprofloxacin; EX, Enrofloxacin; NX, Norfloxacin; AMP, Ampicillin; TE, Tetracycline; S, Streptomycin; GEN, Gentamicin; C, Chloramphenicol; E, Erythromycin; A, Air; F, Feces; Io, Internal organs; T, Trachea; I, Intestine; L, Liver; Lu, Lung; Y, Egg yolk. 
A total of 16 antibiotic resistance patterns were observed among the APEC isolates. Among the antibiotypes, resistance pattern no. 1 (AMP, TE, C, E) was the most prevalent (30.5\%), followed by pattern no. 9 (CIP, EX, NX, AMP, TE, C, E) in 16.7\% of the isolates and pattern no. 12 (CIP, EX, NX, $\mathrm{AMP}, \mathrm{TE}, \mathrm{S}, \mathrm{C}, \mathrm{E}$ ) in $11.1 \%$ of the isolates. One isolate (Io-Lu2) showed resistance to nine antibiotics (seven classes of antimicrobials) of the ten tested.

\subsection{Pairwise Correlation between Resistance to Antimicrobials}

Statistical analysis revealed that norfloxacin and ciprofloxacin resistance showed the strongest significant correlation (Spearman's rank correlation coefficient, $\rho=0.8315$ with $p$-value $=0.0000$ ), followed by a significant correlation between enrofloxacin and ciprofloxacin resistance $(\rho=0.6625$ with $p$-value $=0.0000)$ and norfloxacin and enrofloxacin resistance $(\rho=0.6203$ with $p$-value $=0.0001)$. The pairwise correlations among the resistance to antimicrobials are represented in Table 6.

Table 6. Pairwise correlations between resistance to antimicrobials of APEC isolated from layer chickens $(n=36)^{\mathrm{AB}}$.

\begin{tabular}{|c|c|c|c|c|c|c|c|c|c|c|}
\hline & CL & CIP & EX & NX & AMP & $\mathrm{TE}$ & $\mathbf{S}$ & C & GEN & $\mathbf{E}$ \\
\hline $\mathrm{CL}$ & 1.0000 & - & - & - & - & - & - & - & - & - \\
\hline CIP & 0.1195 & 1.0000 & - & - & - & - & - & - & - & - \\
\hline $\mathrm{EX}$ & 0.2345 & $0.6625 *$ & 1.0000 & - & - & - & - & - & - & - \\
\hline $\mathrm{NX}$ & 0.1383 & 0.8315 * & $0.6203 *$ & 1.0000 & - & - & - & - & - & - \\
\hline AMP & - & - & - & - & - & - & - & - & - & - \\
\hline $\mathrm{TE}$ & - & - & - & - & - & - & - & - & - & - \\
\hline S & -0.0625 & 0.1195 & 0.2132 & 0.1581 & - & - & 1.0000 & - & - & - \\
\hline C & - & - & - & - & - & - & - & - & - & - \\
\hline GEN & 0.1136 & 0.0136 & -0.0823 & 0.0359 & - & - & -0.1136 & - & 1.0000 & - \\
\hline $\mathrm{E}$ & - & - & - & - & - & - & - & - & - & - \\
\hline
\end{tabular}

Here, $\left(^{*}\right)$ indicates a significant correlation with a $p$ value less than or equal to $0.00625(p \leq 0.00625) .{ }^{A} \mathrm{CL}$, Colistin; CIP, Ciprofloxacin; EX, Enrofloxacin; NX, Norfloxacin; AMP, Ampicillin; TE, Tetracycline; S, Streptomycin; C, Chloramphenicol; GEN, Gentamicin; E, Erythromycin. ${ }^{B}$ Spearman rank correlation, with a Bonferroni correction $(\alpha / 8)$ to adjust for multiple comparisons, was performed to determine the pairwise correlation between the resistance to antibiotics.

\section{Discussion}

APEC-associated avian colibacillosis has a significant impact on the poultry industry. APEC are also important from the public health point of view [23]. Previous studies have indicated avian colibacillosis as a prominent disease of commercial chickens [17,21]. Globally, poultry industries are being confronted by enormous economic losses due to the dramatic impact of the disease [41]. To control colibacillosis, multiple antimicrobials have been used indiscriminately, especially in middleand low-income countries including Bangladesh, contributing to the development and spread of AMR. The subsequent selection of MDR strains has generated serious challenges in terms of public health. Sustainable development goals (SDGs) are affected by AMR, especially in targeting hunger, poverty, malnutrition, health, and economic growth [24]. Thus, investigations of APEC strains with regard to virulence genes and AMR profiles may help to curtail their hazardous effects.

In this study, the overall prevalence of E. coli was found to be $82.8 \%$. This is in agreement with Hadiujjaman et al. [42] and Islam et al. [43], who reported the E. coli prevalence in layers to be $80 \%$ and 85\% in Bangladesh. Al Azad et al. [44] reported an isolation rate of E. coli of 100\% in poultry, which is rather higher than ours. However, the observed variation in the prevalence of E. coli in poultry farms may be linked with differences in isolation methods, geographic locations, hygienic practices, sanitation, and other management practices in farms. A significantly higher occurrence of E. coli in 
the fecal samples (100\%) than in the air from inside poultry shades $(67.74 \%)$ and internal organs of layer birds $(80.56 \%)$ is justified, as E. coli is part of the normal microbiota of the digestive tract. Several studies from home and abroad have also detected E. coli in internal organs [17,45-47], the air from inside poultry shades [48], and feces [49].

The current study is chiefly concerned with APEC, which is determined by virulence gene detection. Previously similar investigations have been undertaken across the globe [40,50,51]. According to Chui et al. [52], virulence genes can be used as molecular markers for the detection of specific groups of pathogens. In this study, APEC strains were identified through the detection of the virulence genes fim $C$, $i u c D$, and papC $[12,53]$. The overall prevalence of APEC was $36.4 \%$, with a significantly higher occurrence in internal organs $(66.7 \%)$ than in feces $(21.9 \%)$ and air $(16.1 \%)$ samples. A high prevalence of APEC in internal organs such as the trachea, lungs liver, intestine, and egg yolk is not unusual since the current study collected these organs from dead birds of sick groups. In addition, APEC-as opportunistic pathogens-usually cause secondary infections in internal organs during the occurrence of infectious bronchitis, Newcastle disease, mycoplasmosis, and others [7]. Previously, several studies had detected APEC in the egg yolk [54], trachea [55], lung epithelia [56], liver [17], and intestine [57]. Although APEC are mostly ExPEC, the presence of APEC in the intestine demonstrates the intestinal colonization of E. coli. According to Dho-Moulin [3], APEC are found in the intestinal microbiota of healthy birds showing no disease symptoms. In fact, intestinal microbiota can act as reservoirs for APEC [57]. Therefore, the fecal presence of APEC is not unusual. We also detected APEC in the air from the inside of poultry shades, which may be associated with the fecal contamination of air [48]. Previously, Obeng et al. [58] and Stromberg et al. [49] recorded the prevalence of APEC in fecal samples from layers and broilers as $10 \%$ and 13\%. Kogovšek et al. [59] revealed an association of APEC virulence genes with air samples. This evidence shows that the APEC strains harbored by commercial chickens and their environment can be transmitted from bird to bird and farm to farm through a variety of ways, including air, feces and utensils used in farms. Moreover, APEC strains are zoonotic in nature, and their presence in air and feces within a farm with which humans are associated is a health issue; hence, they pose a great risk for human health [23].

The present study showed that the APEC-associated gene fim C was significantly more prevalent (97.2\%) than $i u c D(58.3 \%)$ and papC (33.3\%). A similar order of prevalence of fim C $(92.7 \%)>i u c D(78.79 \%)$ $>\operatorname{papC}(22.73 \%)$ and fimC $(96.97 \%)>i u c D(82.7 \%)>\operatorname{papC}(30 \%)$ was reported by Janßen et al. [12] and Paixao et al. [8], respectively. In addition, the higher prevalence of fimC compared to that of other virulence genes has been detected in different countries-e.g., $93.6 \%$ fim C, $70.8 \%$ iucD, and $6.5 \%$ papC in China [60]; 95\% fim C, 71.65\% iucD, and 36.65\% papC in Japan [61]; 94.87\% fimC and 8.69\% for both iucD and papC in Italy [62]; and 92\% fim C, 72\% iucD, and 48\% papC in Pakistan [63]. However, both fimC and papC are responsible for the adhesion of E. coli to cells $[8,15]$. The fim $C$ gene plays a greater role in adhesion. The presence of $i u c D$ promotes the survival of APEC, as the gene is associated with iron, which is essential for E.coli survival [64]. Two or more APEC-associated virulence genes were detected at $30.5 \%$ among the E. coli isolated in this study (25/82), while $10 \%$ of the isolates had the same pattern as that identified by Obeng et al. [58]. The detection of virulence genes singly or in combination from clinical samples shows that fimC, $i u c D$, and papC are pivotal virulence genes of APEC strains.

Antimicrobials are widely used as a primary measure to control APEC infections and reduce economic losses globally, particularly in the developing world, including Bangladesh. The antibiotics used in the farm themselves act as a selective pressure for the development of antibiotic resistance in bacteria [65], which has a negative impact on public health [21,66]. We found all the APEC isolates to be resistant to ampicillin and tetracycline, and a high prevalence of resistance to chloramphenicol, erythromycin, enrofloxacin, norfloxacin, and ciprofloxacin. A 100\% resistance of APEC isolates to ampicillin and tetracycline was also reported by Awad et al. [67]. In addition, Subedi et al. [17] and Ozawa et al. [68] reported higher percentages of APEC resistance to ampicillin, tetracycline, ciprofloxacin, and norfloxacin. For a long time, tetracycline has been used as a growth enhancer and a therapeutic agent in livestock production [69]; hence, the high level of resistance observed in this 
study is not surprising. In Bangladesh, tetracycline, and ampicillin have been used extensively to treat diseases in animals and humans (personal communication). However, the most alarming finding from our study is that all the APEC isolates were MDR in class. Similar findings were recorded in E. coli in poultry in Bangladesh [30,70-72], though the E. coli isolates had not been characterized as APEC. Subedi et al. [17] recorded 94\% of APEC isolates as MDR in Nepal, similar to our findings. In South Korea, 94.1\% of isolates were MDR in 2000-2005 [73], and 80.3\% of APEC isolates were shown to be MDR in China [74]. Furthermore, several significant correlations among three fluoroquinolone class of antibiotics were identified in our current study. This finding is important, as researchers have strongly indicated that pairwise correlation can be linked with the development of resistance against other used antimicrobials [40].

Colistin is a last-resort antibiotic drug and should not be used for animal production. In our study, $11.1 \%$ of the isolates were resistant to colistin. Poultry are recognized as one of the major reservoirs and transmitters of colistin resistance [75]. Sobur et al. [32] reported colistin-resistant E. coli in poultry in Bangladesh, but these findings are much more frequent in China [76]. The indiscriminate use of antimicrobials is linked to a high prevalence of MDR strains in E. coli and in other enterobacteria [77]. The MDR and colistin-resistant APEC detected from layer birds and their surrounding air in the current study warn of serious health hazards for working personnel in farms and call for a further health study.

\section{Conclusions}

This is the first study in Bangladesh describing the detection of APEC in layers using molecular methods. In addition to internal organs, fecal samples and air samples were also found to carry APEC. Interestingly, all the APEC isolates were MDR in nature. Our current findings demonstrate that layer birds are potential reservoirs of antibiotic-resistant APEC, posing high public health risks to people who are exposed to them directly or indirectly. Based on importance, further studies should be employed to comprehend the dynamics and genetic diversity of antibiotic-resistant APEC associated with poultry, poultry farms, and their surroundings. However, considering the zoonotic significance, we propose the routine screening of APEC, targeting their virulence genes, for the early detection of avian colibacillosis and, hence, the protection of human health. Furthermore, the prevention of antimicrobial misuse, application of effective biosecurity measures, and adaptation of the one health approach are exigent measures for reducing AMR-related hazards.

Author Contributions: M.T.R. designed the study, secured the funds, and supervised the whole work. S.I. collected the samples and carried out the laboratory work. M.A.S., M.T., and M.S.I. also contributed in the laboratory work. S.I., M.S.I., and M.A.S. wrote the original draft of the manuscript. S.I., M.A.S., and M.S.I. did the data analysis and interpretation. M.F.R.K. and M.B.R. participated in the supervision and revision of the manuscript. S.I., M.S.I., M.A.S., and M.T.R. critically reviewed and edited the manuscript. All authors have read and agreed to this final published version of the manuscript.

Funding: We are deeply grateful to the Ministry of Education, Government of Bangladesh for funding the research project (Project No. LS2018686).

Acknowledgments: We are deeply grateful to the poultry farm owners for giving access to the samples. The authors would also like to thank the Department of Microbiology and Hygiene, Faculty of Veterinary Science Bangladesh Agricultural University, Mymensingh for the support during the research.

Conflicts of Interest: The authors declare that they have no competing interest. The funders had no role in the study design, data collection and analysis, manuscript preparation, or decision to publish the manuscript.

\section{References}

1. MOFL, “Annul Report 2018-2019”, Ministry of Fisheries and Livestock, Bangladesh 2019. Available online: http://bbs.gov.bd/site/page/dc2bc6ce-7080-48b3-9a04-73cec782d0df/- (accessed on 28 April 2020).

2. Alam, S.B.; Mahmud, M.; Akter, R.; Hasan, M.; Sobur, A.; Nazir, K.H.M.; Noreddin, A.; Rahman, T.; El Zowalaty, M.E.; Rahman, M. Molecular detection of multidrug resistant salmonella species isolated from broiler farm in bangladesh. Pathogens 2020, 9, 201. [CrossRef] [PubMed]

3. Dho-Moulin, M.; Fairbrother, J.M. Avian pathogenic Escherichia coli (APEC). Vet. Res. 1999, 30, $299-316$. 
4. Kim, Y.B.; Yoon, M.Y.; Ha, J.S.; Seo, K.W.; Noh, E.B.; Son, S.H.; Lee, Y.J. Molecular characterization of avian pathogenic Escherichia coli from broiler chickens with colibacillosis. Poult. Sci. 2020, 99, 1088-1095. [CrossRef]

5. Schouler, C.; Schaeffer, B.; Brée, A.; Mora, A.; Dahbi, G.; Biet, F.; Oswald, E.; Mainil, J.; Blanco, J.; Moulin-Schouleur, M. Diagnostic strategy for identifying avian pathogenic Escherichia coli based on four patterns of virulence genes. J. Clin. Microbiol. 2012, 50, 1673-1678. [CrossRef]

6. Ewers, C.; Janßen, T.; Kießling, S.; Philipp, H.C.; Wieler, L.H. Molecular epidemiology of avian pathogenic Escherichia coli (APEC) isolated from colisepticemia in poultry. Vet. Microbiol. 2004, 104, 91-101. [CrossRef]

7. Oh, J.Y.; Kang, M.S.; Kim, J.M.; An, B.K.; Song, E.A.; Kim, J.Y.; Shin, E.G.; Kim, M.J.; Kwon, J.H.; Kwon, Y.K. Characterization of Escherichia coli isolates from laying hens with colibacillosis on 2 commercial egg-producing farms in Korea. Poult. Sci. 2011, 90, 1948-1954. [CrossRef] [PubMed]

8. Paixao, A.C.; Ferreira, A.C.; Fontes, M.; Themudo, P.; Albuquerque, T.; Soares, M.C.; Fevereiro, M.; Martins, L.; de Sá, M.I.C. Detection of virulence-associated genes in pathogenic and commensal avian Escherichia coli isolates. Poult. Sci. 2016, 95, 1646-1652. [CrossRef]

9. Redweik, G.A.; Stromberg, Z.R.; Van Goor, A.; Mellata, M. Protection against avian pathogenic Escherichia coli and Salmonella Kentucky exhibited in chickens given both probiotics and live Salmonella vaccine. Poult. Sci. 2020, 99, 752-762. [CrossRef] [PubMed]

10. Yogaratnam, V. Analysis of the causes of high rates of carcase rejection at a poultry processing plant. Vet. Rec. 1995, 137, 215-217. [CrossRef] [PubMed]

11. Al-Kandari, F.; Woodward, M.J. Genotypic and phenotypic diversity differences of presumptive commensal and avian pathogenic E. coli. Br. Poult. Sci. 2019, 60, 79-86. [CrossRef] [PubMed]

12. Janßen, T.; Schwarz, C.; Preikschat, P.; Voss, M.; Philipp, H.C.; Wieler, L.H. Virulence-associated genes in avian pathogenic Escherichia coli (APEC) isolated from internal organs of poultry having died from colibacillosis. Int. J. Med. Microbiol. 2001, 291, 371-378. [CrossRef]

13. Ghunaim, H.; Abu-Madi, M.A.; Kariyawasam, S. Advances in vaccination against avian pathogenic Escherichia coli respiratory disease: Potentials and limitations. Vet. Microbiol. 2014, 172, 13-22. [CrossRef] [PubMed]

14. Huja, S.; Oren, Y.; Trost, E.; Brzuszkiewicz, E.; Biran, D.; Blom, J.; Goesmann, A.; Gottschalk, G.; Hacker, J.; Ron, E.Z.; et al. Genomic avenue to avian colisepticemia. MBio 2015, 6, e01681-14. [CrossRef] [PubMed]

15. Zakariazadeh, N.; Shayegh, J.; Ghorbani, A. Phylogenetic typing and virulence gene profile of pathogenic and commensal avian Escherichia coli in Iran: A notable finding. Comp. Clin. Pathol. 2019, 28, 525-530. [CrossRef]

16. Mohamed, L.; Ge, Z.; Yuehua, L.; Yubin, G.; Rachid, K.; Mustapha, O.; Junwei, W.; Karine, O. Virulence traits of avian pathogenic (APEC) and fecal (AFEC) E. coli isolated from broiler chickens in Algeria. Trop. Anim. Health Prod. 2018, 50, 547-553. [CrossRef] [PubMed]

17. Subedi, M.; Luitel, H.; Devkota, B.; Bhattarai, R.K.; Phuyal, S.; Panthi, P.; Shrestha, A.; Chaudhary, D.K. Antibiotic resistance pattern and virulence genes content in avian pathogenic Escherichia coli (APEC) from broiler chickens in Chitwan, Nepal. BMC Vet. Res. 2018, 14, 113. [CrossRef]

18. Wang, J.; Tang, P.; Tan, D.; Wang, L.; Zhang, S.; Qiu, Y.; Dong, R.; Liu, W.; Huang, J.; Chen, T.; et al. The pathogenicity of chicken pathogenic Escherichia coli is associated with the numbers and combination patterns of virulence-associated genes. Open J. Vet. Med. 2015, 5, 243. [CrossRef]

19. Vandekerchove, D.; Vandemaele, F.; Adriaensen, C.; Zaleska, M.; Hernalsteens, J.P.; De Baets, L.; Butaye, P.; Van Immerseel, F.; Wattiau, P.; Laevens, H.; et al. Virulence-associated traits in avian Escherichia coli: Comparison between isolates from colibacillosis-affected and clinically healthy layer flocks. Vet. Microbiol. 2005, 108, 75-87. [CrossRef] [PubMed]

20. Nie, W.; Wang, J.; Xu, J.; Yao, L.; Qiao, D.; Xue, F.; Tang, F.; Chen, W. A molecule capturer analysis system for visual determination of avian pathogenic Escherichia coli serotype O78 using a lateral flow assay. Microchim. Acta 2020, 187, 1-7. [CrossRef] [PubMed]

21. Mellata, M. Human and avian extraintestinal pathogenic Escherichia coli: Infections, zoonotic risks, and antibiotic resistance trends. Foodborne Pathog. Dis. 2013, 10, 916-932. [CrossRef]

22. Manges, A.R.; Johnson, J.R. Food-borne origins of Escherichia coli causing extraintestinal infections. Clin. Infect. Dis. 2012, 55, 712-719. [CrossRef] [PubMed] 
23. Tivendale, K.A.; Logue, C.M.; Kariyawasam, S.; Jordan, D.; Hussein, A.; Li, G.; Wannemuehler, Y.; Nolan, L.K. Avian-pathogenic Escherichia coli strains are similar to neonatal meningitis E. coli strains and are able to cause meningitis in the rat model of human disease. Infect. Immun. 2010, 78, 3412-3419. [CrossRef] [PubMed]

24. Clifford, K.; Darash, D.; da Costa, C.P.; Meyer, H.; Islam, M.T.; Meyer, H.; Klohe, K.; Winklerc, A.; Rahman, M.T.; Islam, M.T.; et al. The threat of antimicrobial resistance opportunities for a technology-integrated One Health approach. Bull. World Health Organ. 2018, 96, 662-664. [CrossRef]

25. Jakobsen, L.; Spangholm, D.J.; Pedersen, K.; Jensen, L.B.; Emborg, H.D.; Agersø, Y.; Aarestrup, F.M.; Hammerum, A.M.; Frimodt-Møller, N. Broiler chickens, broiler chicken meat, pigs and pork as sources of ExPEC related virulence genes and resistance in Escherichia coli isolates from community-dwelling humans and UTI patients. Int. J. Food Microbiol. 2010, 142, 264-272. [CrossRef] [PubMed]

26. WHO. Antimicrobial Resistance in the Food Chain, Retrieved 2019. Available online: https://www.who.int/ foodsafety/areas_work/antimicrobial-resistance/amrfoodchain/en/ (accessed on 28 April 2020).

27. Ahmed, A.M.; Shimamoto, T.; Shimamoto, T. Molecular characterization of multidrug-resistant avian pathogenic Escherichia Coli isolated from septicemic broilers. Int. J. Med. Microbiol. 2013, 303, 475-483. [CrossRef]

28. Ozaki, H.; Matsuoka, Y.; Nakagawa, E.; Murase, T. Characteristics of Escherichia coli isolated from broiler chickens with colibacillosis in commercial farms from a common hatchery. Poult. Sci. 2017, 96, 3717-3724. [CrossRef]

29. Hossain, M.T.; Siddique, M.P.; Hossain, F.M.A.; Zinnah, M.A.; Hossain, M.M.; Alam, M.K.; Rahman, M.T.; Choudury, K.A. Isolation, identification, toxin profile and antibiogram of Escherichia coli isolated from broilers and layers in Mymensingh district of Bangladesh. Bangladesh J. Vet. Med. 2008, 6, 1-5. [CrossRef]

30. Akond, M.A.; Alam, S.; Hassan, S.M.R.; Shirin, M. Antibiotic resistance of Escherichia coli isolated from poultry and poultry environment of Bangladesh. Int. J. Food Saf. 2009, 11, 19-23.

31. Parvej, M.S.; Alam, M.A.; Shono, M.; Zahan, M.N.; Parvez, M.M.M.; Ansari, W.K.; Jowel, M.S.; Uddin, M.S.; Kage-Nakadai, E.; Rahman, M.T.; et al. Prevalence of virulence genes of diarrheagenic Escherichia coli in fecal samples obtained from cattle, poultry and diarrheic patients in Bangladesh. Jpn. J. Infect. Dis. 2020, 73, 76-82. [CrossRef]

32. Sobur, M.A.; Ievy, S.; Haque, Z.F.; Nahar, A.; Zaman, S.B.; Rahman, M.T. Emergence of colistin-resistant Escherichia coli in poultry, house flies, and pond water in Mymensingh, Bangladesh. J. Adv. Vet. Anim. Res. 2019, 6, 50-53.

33. Mbamalu, O.; Uebel, R.; Meki, B. Control of airborne microbes in a poultry setting using Dioxy MP 14. Braz. J. Poult. Sci. 2015, 17, 77-86. [CrossRef]

34. Bergey, D.H.; Buchanan, R.E.; Gibbons, N.E. Bergey's Manual of Determinative Bacteriology, 8th ed.; American Society for Microbiology: Washington, DC, USA, 1974; pp. 966-1097.

35. Sobur, A.; Haque, Z.F.; Sabuj, A.A.; Ievy, S.; Rahman, A.T.; El Zowalaty, M.E.; Rahman, T. Molecular detection of multidrug and colistin-resistant Escherichia coli isolated from house flies in various environmental settings. Future Microbiol. 2019, 14, 847-858. [CrossRef]

36. Mahmud, S.; Nazir, K.N.H.; Rahman, M.T. Prevalence and molecular detection of fluoroquinolone-resistant genes ( $q n r A$ and $q n r S$ ) in Escherichia coli isolated from healthy broiler chickens. Vet. World 2018, 11, 1720-1724. [CrossRef] [PubMed]

37. Bayer, A.W.; Kirby, W.M.; Sherris, J.C.; Turck, M. Antibiotic susceptibility testing by a standardized single disc method. Am. J. Clin. Pathol. 1966, 45, 493-496. [CrossRef]

38. CLSI. Performance Standards for Antimicrobial Susceptibility Testing, 26th ed.; CLSI Supplement M100s; Clinical and Laboratory Standards Institute: Wayne, PA, USA, 2016.

39. Sweeney, M.T.; Lubbers, B.V.; Schwarz, S.; Watts, J.L. Applying definitions for multidrug resistance, extensive drug resistance and pandrug resistance to clinically significant livestock and companion animal bacterial pathogens. J. Antimicrob. Chemother. 2018, 73, 1460-1463. [CrossRef]

40. Varga, C.; Brash, M.L.; Slavic, D.; Boerlin, P.; Ouckama, R.; Weis, A.; Petrik, M.; Philippe, C.; Barham, M.; Guerin, M.T. Evaluating virulence-associated genes and antimicrobial resistance of avian pathogenic Escherichia coli isolates from broiler and broiler breeder chickens in Ontario, Canada. Avian Dis. 2018, 62, 291-299. [CrossRef] 
41. Logue, C.; Newman, D.; Nolan, L.; Barbieri, N. Characterizing avian pathogenic Escherichia coli from diagnostic cases in Georgia, USA-comparison of gene profiles with tissue of isolation. Acc. Microbiol. 2019, 1, 716. [CrossRef]

42. Hadiujjaman, M.; Rahman, M.M.; Ahasan, M.D.; Banu, M.A.; Khatun, M.M.; Islam, M.A. Isolation and identification of Escherichia coli from apparently healthy chicken of selected areas of Bangladesh. Int. J. Nat. Soc. Sci. 2016, 3, 15-23.

43. Islam, K.; Kabir, S.L.; Haque, A.K.M.Z.; Sarker, Y.A.; Sikder, M.H. Molecular detection and characterization of Escherichia coli, Salmonella spp. and Campylobacter spp. isolated from broiler meat in Jamalpur, Tangail, Netrokona and Kishoreganj districts of Bangladesh. Afr. J. Microbiol. Res. 2018, 12, 761-770.

44. Al Azad, M.; Rahman, A.; Rahman, M.; Amin, R.; Begum, M.; Ara, I.; Fries, R.; Husna, A.; Khairalla, A.S.; Badruzzaman, A.T.M.; et al. Susceptibility and multidrug resistance patterns of Escherichia coli isolated from cloacal swabs of live broiler chickens in Bangladesh. Pathogens 2019, 8, 118. [CrossRef]

45. Tonu, N.S.; Sufian, M.A.; Sarker, S.; Kamal, M.M.; Rahman, M.H.; Hossain, M.M. Pathological study on colibacillosis in chickens and detection of Escherichia coli by PCR. Bangladesh J. Vet. Med. 2011, 9, 17-25. [CrossRef]

46. Khaton, R.; Haider, M.G.; Paul, P.K.; Das, P.M.; Hossain, M.M. Colibacillosis in commercial chickens in Bangladesh. Bangladesh Vet. 2008, 25, 17-24. [CrossRef]

47. Ibrahim, R.A.; Cryer, T.L.; Lafi, S.Q.; Basha, E.A.; Good, L.; Tarazi, Y.H. Identification of Escherichia coli from broiler chickens in Jordan, their antimicrobial resistance, gene characterization and the associated risk factors. BMC Vet. Res. 2019, 15, 159. [CrossRef]

48. Duan, H.; Chai, T.; Cai, Y.; Zhong, Z.; Yao, M.; Zhang, X. Transmission identification of Escherichia coli aerosol in chicken houses to their environments using ERIC-PCR. Sci. China Life Sci. 2008, 51, 164-173. [CrossRef]

49. Stromberg, Z.R.; Johnson, J.R.; Fairbrother, J.M.; Kilbourne, J.; Van Goor, A.; Curtiss, R., III; Mellata, M. Evaluation of Escherichia coli isolates from healthy chickens to determine their potential risk to poultry and human health. PLoS ONE 2017, 12, e0180599. [CrossRef] [PubMed]

50. Azam, M.; Mohsin, M.; Saleemi, M.K. Virulence-associated genes and antimicrobial resistance among avian pathogenic Escherichia coli from colibacillosis affected broilers in Pakistan. Trop. Anim. Health Prod. 2019, 51, 1259-1265. [CrossRef]

51. Handrova, L.; Kmet, V. Antibiotic resistance and virulence factors of Escherichia coli from eagles and goshawks. J. Environ. Sci. Health B 2019, 54, 605-614. [CrossRef] [PubMed]

52. Chui, L.; Couturier, M.R.; Chiu, T.; Wang, G.; Olson, A.B.; McDonald, R.R.; Antonishyn, N.A.; Horsman, G.; Gilmour, M.W. Comparison of Shiga toxin-producing Escherichia coli detection methods using clinical stool samples. J. Mol. Diagn. 2010, 12, 469-475. [CrossRef] [PubMed]

53. Sgariglia, E.; Mandolini, N.A.; Napoleoni, M.; Medici, L.; Fraticelli, R.; Conquista, M.; Gianfelici, P.; Staffolani, M.; Fisichella, S.; Capuccella, M.; et al. Antibiotic resistance pattern and virulence genes in avian pathogenic Escherichia coli (APEC) from different breeding systems. Vet. Ital. 2019, 55, 27-33.

54. Kemmett, K.; Williams, N.J.; Chaloner, G.; Humphrey, S.; Wigley, P.; Humphrey, T. The contribution of systemic Escherichia coli infection to the early mortalities of commercial broiler chickens. Avian Pathol. 2014, 43, 37-42. [CrossRef]

55. Dho, M.; Lafont, J.P. Escherichia coli colonization of the trachea in poultry: Comparison of virulent and avirulent strains in gnotoxenic chickens. Avian Dis. 1982, 26, 787-797. [CrossRef] [PubMed]

56. Mol, N.; Peng, L.; Esnault, E.; Quéré, P.; Haagsman, H.P.; Veldhuizen, E.J. Avian pathogenic Escherichia coli infection of a chicken lung epithelial cell line. Vet. Immunol. Immunopathol. 2019, 210, 55-59. [CrossRef] [PubMed]

57. Ewers, C.; Antão, E.M.; Diehl, I.; Philipp, H.C.; Wieler, L.H. Intestine and environment of the chicken as reservoirs for extraintestinal pathogenic Escherichia coli strains with zoonotic potential. Appl. Environ. Microbiol. 2009, 75, 184-192. [CrossRef] [PubMed]

58. Obeng, A.S.; Rickard, H.; Ndi, O.; Sexton, M.; Barton, M. Antibiotic resistance, phylogenetic grouping and virulence potential of Escherichia coli isolated from the faeces of intensively farmed and free range poultry. Vet. Microbiol. 2012, 154, 305-315. [CrossRef]

59. Kogovšek, P.; Ambrožič-Avguštin, J.; Dovč, A.; Dreo, T.; Hristov, H.; Krapež, U.; Ravnikar, M.; Slavec, B.; Lotrič, M.; Žel, J.; et al. Loop-mediated isothermal amplification: Rapid molecular detection of virulence genes associated with avian pathogenic Escherichia coli in poultry. Poult. Sci. 2019, 98, 1500-1510. [CrossRef] 
60. Jin, W.J.; Zheng, Z.M.; Zhang, Y.Z.; Qin, A.J.; Shao, H.X.; Liu, Y.L.; Jiao, W.A.N.G.; Wang, Q.Q. Distribution of virulence-associated genes of avian pathogenic Escherichia coli isolates in China. Agr. Sci. China 2008, 7, 1511-1515. [CrossRef]

61. Kawano, M.; Yaguchi, K.; Osawa, R. Genotypic analyses of Escherichia coli isolated from chickens with colibacillosis and apparently healthy chickens in Japan. Microbiol. Immunol. 2006, 50, 961-966. [CrossRef]

62. Camarda, A.; Circella, E.; Giovanardi, D.; Pennelli, D.; Battista, P.; Campagnari, E.; Bruni, G.; Tagliabue, S. Avian Pathogenic Escherichia coli in Audouin gulls (Larus audouinii) Could they affect the surviving of the bird colonies? Ital. J. Anim. 2007, 6, 317-320. [CrossRef]

63. Jan, A.W.; Javed, M.T.; Lone, S.Q.; Aslam, M.S.; Javed, A. Association of six selected pathogenicity genes of Escherichia coli with gross and histopathological lesions in broiler chickens from field cases. Pak. J. Agri. Sci. 2018, 55, 433-440.

64. Gao, Q.; Wang, X.; Xu, H.; Xu, Y.; Ling, J.; Zhang, D.; Gao, S.; Liu, X. Roles of iron acquisition systems in virulence of extraintestinal pathogenic Escherichia coli: Salmochelin and aerobactin contribute more to virulence than heme in a chicken infection model. BMC Microbiol. 2012, 12, 143. [CrossRef]

65. Wright, G.D. The antibiotic resistome: The nexus of chemical and genetic diversity. Nat. Rev. Microbiol. 2007, 5, 175-186. [CrossRef] [PubMed]

66. Miles, T.D.; McLaughlin, W.; Brown, P.D. Antimicrobial resistance of Escherichia coli isolates from broiler chickens and humans. BMC Vet. Res. 2006, 2, 7. [CrossRef] [PubMed]

67. Awad, A.M.; El-Shall, N.A.; Khalil, D.S.; El-Hack, A.; Mohamed, E.; Swelum, A.A.; Mahmoud, A.H.; Ebaid, H.; Komany, A.; Sammour, R.H.; et al. Incidence, pathotyping, and antibiotic susceptibility of avian pathogenic Escherichia coli among diseased broiler chicks. Pathogens 2020, 9, 114. [CrossRef] [PubMed]

68. Ozawa, M.; Harada, K.; Kojima, A.; Asai, T.; Sameshima, T. Antimicrobial susceptibilities, serogroups, and molecular characterization of avian pathogenic Escherichia coli isolates in Japan. Avian Dis. 2008, 52, 392-397. [CrossRef] [PubMed]

69. Cardoso, M.O.; Ribeiro, A.R.; Santos, L.R.D.; Pilotto, F.; de Moraes, H.L.; Salle, C.T.P.; Rocha, S.L.D.S.; Nascimento, V.P.D. Antibiotic resistance in Salmonella enteritidis isolated from broiler carcasses. Braz. J. Microbiol. 2006, 37, 368-371. [CrossRef]

70. Sarker, M.S.; Mannan, M.S.; Ali, M.Y.; Bayzid, M.; Ahad, A.; Bupasha, Z.B. Antibiotic resistance of Escherichia coli isolated from broilers sold at live bird markets in Chattogram, Bangladesh. J. Adv. Vet. Anim. Res. 2019, 6, 272-277. [CrossRef]

71. Azad, M.A.R.A.; Amin, R.; Begum, M.I.A.; Fries, R.; Lampang, K.N.; Hafez, H.M. Prevalence of antimicrobial resistance of Escherichia coli isolated from broiler at Rajshahi region, Bangladesh. Br. J. Biomed. Multidisc. Res. 2017, 1, 6-12.

72. Bashar, T.; Rahman, M.; Rabbi, F.A.; Noor, R.; Rahman, M.M. Enterotoxin profiling and antibiogram of Escherichia coli isolated from poultry feces in Dhaka district of Bangladesh. Stamford J. Microbiol. 2011, 1, 51-57. [CrossRef]

73. Kim, T.E.; Jeong, Y.W.; Cho, S.H.; Kim, S.J.; Kwon, H.J. Chronological study of antibiotic resistances and their relevant genes in Korean avian pathogenic Escherichia Coli isolates. J. Clin. Microbiol. 2007, 45, 3309-3315. [CrossRef]

74. Dou, X.; Gong, J.; Han, X.; Xu, M.; Shen, H.; Zhang, D.; Zhuang, L.; Liu, J.; Zou, J. Characterization of avian pathogenic Escherichia coli isolated in eastern China. Gene 2016, 576, 244-248. [CrossRef]

75. Hoelzer, K.; Wong, N.; Thomas, J.; Talkington, K.; Jungman, E.; Coukell, A. Antimicrobial drug use in food-producing animals and associated human health risks: What, and how strong, is the evidence? BMC Vet. Res. 2017, 13, 211. [CrossRef] [PubMed]

76. Zhao, X.; Liu, Z.; Zhang, Y.; Yuan, X.; Hu, M.; Liu, Y. Prevalence and molecular characteristics of avian-origin mcr-1-harboring Escherichia coli in Shandong province, China. Front. Microbiol. 2020, 11, 255. [CrossRef] [PubMed]

77. van den Bogaard, A.E.; Stobberingh, E.E. Epidemiology of resistance to antibiotics: Links between animals and humans. Int. J. Antimicrob. Agents 2000, 14, 327-335. [CrossRef]

(C) 2020 by the authors. Licensee MDPI, Basel, Switzerland. This article is an open access article distributed under the terms and conditions of the Creative Commons Attribution (CC BY) license (http://creativecommons.org/licenses/by/4.0/). 